\title{
APLICAÇÃO DE PROCESSO DE COAGULAÇÃO/FLOCULAÇÃO/FILTRAÇÃO COM MEMBRANAS PARA A PRODUÇÃO DE ÁGUA PARA CONSUMO HUMANO
}

\author{
G. S. MADRONA ${ }^{1}$, G. G. PIERETTI ${ }^{1}$, J. L. ANTIGO ${ }^{1}$, M. R. S. SCAPIM ${ }^{1}$, K. C. \\ VALVERDE $^{2}$, R. BERGAMASCO ${ }^{2}$ \\ ${ }^{1}$ Universidade Estadual de Maringá, Departamento de Engenharia de Alimentos \\ ${ }^{2}$ Universidade Estadual de Maringá, Departamento de Engenharia Química \\ E-mail para contato: gsmadrona@uem.br
}

\begin{abstract}
RESUMO - Entre os coagulantes naturais usados no tratamento de água, destaca-se a polpa da semente de Moringa oleifera Lam (MO) e como tecnologia surge à utilização de processos combinados (coagulação/floculação/filtração com membranas - CFF) em função de suas vantagens. O presente trabalho avaliou a eficiência da MO no processo de CFF. Foram utilizadas membranas 0,1 e $0,2 \mu \mathrm{m}$ e pressão de 1 bar, os ensaios foram conduzidos utilizando somente água bruta, solução de Moringa extraída com água e com solução salina $(\mathrm{KCl} 1 \mathrm{M})$. Os parâmetros avaliados foram cor, turbidez, DQO, $\mathrm{pH}$, compostos com absorção em UV e coliformes. Avaliou-se ainda o fluxo e entupimento da membrana. Os resultados encontrados demonstram que o processo de CFF é eficiente para se obter água com o devido padrão de potabilidade. Os menores entupimentos aconteceram no processo combinado, utilizando-se a extração com solução salina. Portanto, a conclusão deste estudo é de que a associação dos processos e a aplicação da MO é uma alternativa viável na obtenção de água para consumo humano.
\end{abstract}

\section{INTRODUÇÃO}

A qualidade da água está diretamente relacionada ao adensamento populacional, e diferentes usos do solo da bacia em questão. A contaminação dos recursos hídricos se constitui em um fator de risco para a saúde da população, especialmente em regiões com condições inadequadas de saneamento e suprimento de água. Essa situação compromete gravemente os corpos d'água, sendo necessária a escolha de uma tecnologia de tratamento adequada. Nesta seleção, deve ser buscada a sustentabilidade do sistema, a fim de garantir que a água tenha quantidade e qualidade satisfatórias e compatíveis com as necessidades da população (Camplesi, 2010).

No processo de tratamento convencional de água aplica-se na etapa de coagulação, agentes coagulantes sintéticos. Entre os principais agentes utilizados atualmente nas estações de tratamento de águas, tem-se o sulfato de alumínio, cloreto férrico, sulfato ferroso, sulfato férrico, policloreto de alumínio, polímeros catiônicos, entre outros. No entanto, tem-se verificado, nos últimos anos, a busca por coagulantes naturais com características próximas aos produtos químicos empregados no processo de coagulação/floculação para o tratamento de água e efluentes (Conceição et al., 2013). 


\section{9 a 22 de outubro de 2014 \\ Florianópolis/SC}

Os coagulantes naturais apresentam várias vantagens em relação aos coagulantes químicos, pois são biodegradáveis, não tóxicos e ainda produzem lodo em menor quantidade e com menores teores de metais quando comparados aos coagulantes químicos (Madrona et al., 2010).

Moringa oleifera Lam é uma árvore pertencente à família das Moringaceae, nativa da Índia e amplamente cultivada nos trópicos (Cardoso et al., 2008). Segundo Ndabigengesere e Narasiah (1996), as sementes de Moringa oleifera Lam (MO) são uma alternativa viável de agente coagulante em substituição aos sais de alumínio, utilizados no tratamento de água. Comparadas com o alumínio, essas sementes não alteram significativamente o $\mathrm{pH}$ e a alcalinidade da água após o tratamento e não causam problemas de corrosão.

Como normalmente não é possível obter água totalmente clarificada apenas por meio do uso da coagulação/floculação e decantação, deve-se fazer uso também da filtração, a fim de remover as partículas suspensas e coloidais que resistem a outros processos de tratamento. Atualmente, tem-se a opção de utilizar diferentes meios filtrantes, incluindo processos com membranas, particularmente a ultra e a microfiltração. De acordo com GUIGUI et al. (2002) a utilização de membranas de ultrafiltração, tem se tornado rapidamente uma alternativa eficiente para o tratamento convencional de produção de água potável, principalmente quando usadas para remoção de turbidez e microrganismos.

Portanto, a utilização de coagulantes naturais nos processos de coagulação e floculação e também o processo combinado de coagulação/floculação/filtração com membranas é uma opção para o tratamento convencional. A adição de coagulantes antes de unidades de ultra ou microfiltração, com ou sem sedimentação, pode aumentar a remoção de matéria orgânica natural e, consequentemente, produzir uma melhor redução dos produtos formados pela desinfecção, diminuindo a adição de produtos químicos no processo de tratamento da água a ser consumida pelos seres humanos.

Neste contexto o objetivo do presente trabalho foi avaliar a eficiência da MO no processo de Coagulação/Floculação/Filtração, utilizando como condições de processo membranas 0,1 e $0,2 \mu \mathrm{m}$, água de turbidez inicial 150 uT e pressão de 1 bar.

\section{MATERIAIS E MÉTODOS}

\subsection{Processo de Coagulação/floculação}

A solução coagulante de MO foi preparada sempre no dia da análise, primeiramente triturou-se no liquidificador $1 \mathrm{~g}$ de polpa de semente com $100 \mathrm{~mL}$ da solução água ou $\mathrm{KCl} 1 \mathrm{Molar}$. Após agitouse a solução durante 30 minutos e filtrou-se a vácuo esta solução (Madrona et al, 2011).

Utilizou-se concentrações de extrato de $\mathrm{MO}$ de 25 a $300 \mathrm{mg} / \mathrm{L}$ no processo de coagulação/floculação e água bruta com 150 uT e um planejamento segundo Nkurunziza et al., (2009), obtendo-se assim as condições otimizadas de processo apresentadas na Tabela 1 para a turbidez avaliada (150 uT). A água bruta utilizada no desenvolvimento da pesquisa foi coletada na 
Companhia de Saneamento do Paraná - SANEPAR, proveniente da bacia do Rio Pirapó - Paraná e foi caracterizada segundo procedimento recomendado pelo Standard Methods (Apha, 1995).

Tabela 1 - Concentração ótima do processo de coagulação/floculação aplicadas no processo combinado de coagulação/ floculação/filtração com membranas

\begin{tabular}{l|lc}
\hline Característica da Água Bruta & Solução coagulante & Concentração (mg/L) \\
\hline Turbidez (150 uT) & Solução extraída com água (SCA) & 25 \\
\cline { 2 - 3 } & Solução extraída com KCl 1 M (SC) & 100
\end{tabular}

Foram realizados ensaios de coagulação/floculação/sedimentação em "jar test" simples, Milan - Modelo JT101/6 de seis provas com regulador de rotação das hastes misturadoras. Foram utilizadas as condições de trabalho para o processo de coagulação/floculação/ sedimentação segundo Cardoso (2008), sendo a velocidade de mistura rápida (100 rpm), o tempo de coagulação (3 minutos), a velocidade de mistura lenta (15 rpm), o tempo de floculação (15 minutos) e o tempo de decantação (60 minutos) e temperatura de $25^{\circ} \mathrm{C}$.

A eficiência do processo foi avaliada por meio de remoção de cor, turbidez e compostos com absorção em UV - 254 nm medidos em espectofotômetro HACH DR/2010, pH e DQO segundo procedimento recomendado pelo Standard Methods (Apha, 1995). A análise de coliformes totais e $E$. coli foi realizada com placa específica para este microorganismo da $3 \mathrm{M}$.

\subsection{Processo de Filtração com membranas}

Convencionou-se trabalhar com valor extremo do planejamento, ou seja, a menor turbidez estudada (150 uT - considerada água com características de cor e turbidez relativamente baixas). Após a otimização dos processos de coagulação e floculação foram realizados ensaios em "jar test", nas condições otimizadas (Tabela 1) e realizou-se então o processo de filtração com duas membranas.

Para tanto, utilizou-se uma unidade de micro-ultrafiltração NETZSCH, pelo princípio da filtração tangencial, na qual foi possível adaptar diferentes tipos de membrana para a filtragem com a troca do "housing" ou suporte da membrana. A primeira membrana era de porosidade de $0,1 \mu \mathrm{m}$, monocanal, a segunda, de porosidade $0,2 \mu \mathrm{m}$, também monocanal. As membranas monocanal possuíam área de filtração de $0,005 \mathrm{~m}^{2}$. A pressão de trabalho escolhida foi de 1 bar, baseando-se em resultados de ensaios preliminares não apresentados neste trabalho. Todo o processo realizado nesta etapa, bem como, os cálculos de fluxo, remoção e entupimento, foram realizados segundo metodologia descrita por Bergamasco et al., (2011).

Para se ter uniformidade nos ensaios fixou-se o volume inicial da alimentação em 20 litros e o tempo de ensaio em 180 minutos. Após cada ensaio de filtração a membrana foi submetida à limpeza. Posteriormente, o fluxo da água de osmose foi medido em temperatura de $25^{\circ} \mathrm{C}$, para verificar se a permeabilidade da membrana havia retornado ao valor inicial após o processo de limpeza. 


\section{RESULTADOS E DISCUSSÃO}

As Tabelas 2 e 3 apresentam os valores obtidos para água de turbidez $150 \mathrm{uT}$, nas membranas de 0,1 e 0,2 $\mu \mathrm{m}$, respectivamente, no processo combinado (coagulação/floculação/ultrafiltração CFU) e apenas na coagulação/floculação para as soluções coagulantes extraídas com água (MSCA) e extraída com sal (MSC) na pressão de 1 bar.

Tabela 2 - Eficiência do processo de ultrafiltração e do processo combinado (coagulação/floculação/ultrafiltração) para os diferentes coagulantes, a 1 bar e membrana $0,1 \mu \mathrm{m}$.

\begin{tabular}{|c|c|c|c|c|c|c|c|c|c|c|}
\hline \multirow[t]{2}{*}{$\begin{array}{l}\text { Parâmetros } \\
\text { analisados }\end{array}$} & $\mathrm{AB}$ & UF & \begin{tabular}{|l|} 
Ef \\
$(\%)$
\end{tabular} & $\mathrm{CF}$ & $\mathrm{CFU}$ & $\begin{array}{l}\text { Ef } \\
(\%)\end{array}$ & $\mathrm{CF}$ & $\mathrm{CFU}$ & \begin{tabular}{|l|} 
Ef \\
$(\%)$
\end{tabular} & \multirow[t]{2}{*}{$\begin{array}{l}\text { Portaria } \\
\text { 2914/MS }\end{array}$} \\
\hline & & \multicolumn{2}{|c|}{ Água Bruta } & \multicolumn{3}{|c|}{ MSCA } & \multicolumn{3}{|c|}{ MSC } & \\
\hline $\begin{array}{l}\text { Cor } \\
\text { Aparente } \\
(\mathrm{uH})\end{array}$ & 515 & $\mathrm{Nd}$ & \begin{tabular}{|l|l}
100,0 \\
\end{tabular} & 326,15 & $\mathrm{Nd}$ & 100,0 & 52,01 & $\mathrm{Nd}$ & 100,0 & 15 \\
\hline $\begin{array}{l}\text { Turbidez } \\
\text { (uT) }\end{array}$ & 150 & 0,6 & 99,50 & 88,23 & 0,1 & 99,91 & 10,28 & 0,5 & 99,59 & $0,5 / 1,0$ \\
\hline $\begin{array}{l}\text { UV-254 nm } \\
\left(\mathrm{cm}^{-1}\right)\end{array}$ & 0,323 & $\mathrm{Nd}$ & 100,0 & 0,308 & 0,001 & 99,69 & 0,071 & $\mathrm{Nd}$ & 100,0 & - \\
\hline $\mathrm{pH}$ & 7,91 & 7,63 & - & 7,43 & 7,34 & - & 7,28 & 6,82 & - & $6,0-9,5$ \\
\hline $\begin{array}{l}\text { Coliformes } \\
\text { Totais } \\
\text { (UFC/mL) }\end{array}$ & 1.500 & $\mathrm{Nd}$ & 99,99 & 500 & $\mathrm{Nd}$ & 99,99 & 500 & $\mathrm{Nd}$ & 99,99 & Ausência \\
\hline $\begin{array}{l}\text { E. coli } \\
\text { (UFC/mL) }\end{array}$ & 150 & $\mathrm{Nd}$ & 99,99 & 50 & $\mathrm{Nd}$ & 99,99 & 50 & $\mathrm{Nd}$ & 99,99 & Ausência \\
\hline $\begin{array}{l}\mathrm{DQO} \\
\left(\mathrm{mgO}^{2} \mathrm{~L}^{-1}\right)\end{array}$ & 8,64 & 0,54 & 93,75 & $\mathrm{Nr}$ & 1,52 & 82,40 & $\mathrm{Nr}$ & 1,68 & 80,55 & - \\
\hline
\end{tabular}

AB- Água Bruta; UF- ultrafiltração; Nd- não detectado; Nr- não realizado; CF - coagulação/floculação; CFU - coagulação/floculação/ultrafiltração; Ef - eficiência de remoção no processo combinado; MSCA solução coagulante extraída com água e MSC - solução coagulante extraída com sal.

Nota-se que em todos os casos apenas o processo de coagulação/floculação (CF) não foi eficaz para remoção dos parâmetros avaliados (Tabelas 2 e 3). Segundo Bawa et al. (2001), a etapa de coagulação/floculação para águas que apresentam baixa turbidez é difícil, pois os flocos formados demonstram dificuldade para decantar. Assim, neste caso, são necessárias etapas posteriores para obtenção de água com padrão de potabilidade (Brasil, 2011). Porém, vale salientar que após este processo $(\mathrm{CF})$, em uma Estação de Tratamento de água convencional, ainda haverá o processo de filtração e no caso do presente estudo, propõe-se o processo de ultrafiltração. Assim, é de fundamental importância a aplicação de uma etapa de filtração no tratamento para obtenção de água potável, quando se utiliza a Moringa oleifera como coagulante.

Em todos os casos avaliados, as remoções de cor foram de $100 \%$, as de turbidez e compostos com absorção em UV-254 nm foram superiores a $99 \%$. A remoção de demanda química de oxigênio 
(DQO) foi efetiva sendo superior a $80 \%$ em todos os ensaios realizados. Obtendo-se assim, água para consumo humano dentro dos padrões de legislação vigente no Brasil (Brasil, 2011).

Tabela 3 - Eficiência do processo de microfiltração e do processo combinado (coagulação/floculação/microfiltração) para os diferentes coagulantes, a 1 bar e membrana 0,2 $\mu$ m.

\begin{tabular}{|c|c|c|c|c|c|c|c|c|c|c|}
\hline \multirow[t]{2}{*}{$\begin{array}{l}\text { Parâmetros } \\
\text { analisados }\end{array}$} & $\mathrm{AB}$ & $\overline{\mathrm{MC}}$ & $\begin{array}{l}\mathrm{Ef} \\
(\%)\end{array}$ & $\overline{\mathrm{CF}}$ & $\overline{\mathrm{CFM}}$ & $\begin{array}{l}\mathrm{Ef} \\
(\%)\end{array}$ & $\overline{\mathrm{CF}}$ & $\overline{C F M}$ & $\begin{array}{l}\mathrm{Ef} \\
(\%)\end{array}$ & \multirow[t]{2}{*}{$\begin{array}{l}\text { Portaria } \\
\text { 2914/MS }\end{array}$} \\
\hline & & \multicolumn{2}{|c|}{ Água Bruta } & \multicolumn{3}{|c|}{ MSCA } & \multicolumn{3}{|c|}{ MSC } & \\
\hline $\begin{array}{l}\text { Cor } \\
\text { Aparente } \\
\text { (uH) }\end{array}$ & 515 & $\mathrm{Nd}$ & 100,00 & 312,2 & $\mathrm{Nd}$ & 100,00 & 48,98 & $\mathrm{Nd}$ & 100,00 & 15 \\
\hline $\begin{array}{l}\text { Turbidez } \\
\text { (uT) }\end{array}$ & 122 & 0,1 & 99,92 & 94,74 & 0,1 & 99,92 & 11,04 & 0,2 & 99,85 & $0,5 / 1,0$ \\
\hline $\begin{array}{l}\text { UV-254 nm } \\
\left(\mathrm{cm}^{-1}\right)\end{array}$ & 0,323 & 0,02 & 97,56 & 0,782 & 0,03 & 96,34 & 0,181 & 0,01 & 98,78 & - \\
\hline $\mathrm{pH}$ & 7,91 & 7,57 & - & 6,25 & 6,38 & - & 6,47 & 6,50 & - & $6,0-9,5$ \\
\hline $\begin{array}{l}\text { Coliformes } \\
\text { Totais } \\
\text { (UFC/mL) }\end{array}$ & 1.500 & $\mathrm{Nd}$ & 99,99 & 400 & $\mathrm{Nd}$ & 99,99 & 350 & $\mathrm{Nd}$ & 99,99 & Ausência \\
\hline $\begin{array}{l}\text { E. coli } \\
\text { (UFC/mL) }\end{array}$ & 150 & $\mathrm{Nd}$ & 99,99 & 100 & $\mathrm{Nd}$ & 99,99 & 180 & $\mathrm{Nd}$ & 99,99 & Ausência \\
\hline $\begin{array}{l}\mathrm{DQO} \\
\left(\mathrm{mgO}^{2} \mathrm{~L}^{-1}\right)\end{array}$ & 8,64 & 1,1 & 81,67 & $\mathrm{Nr}$ & 0,5 & 91,67 & $\mathrm{Nr}$ & 0,84 & 86,00 & - \\
\hline
\end{tabular}

AB- Água Bruta; MC- microfiltração; Nd- não detectado; Nr- não realizado; $\mathrm{CF}$ - coagulação/floculação; CFM - coagulação/floculação/microfiltração; Ef - eficiência de remoção no processo combinado; MSCA - solução coagulante extraída com água e MSC - solução coagulante extraída com sal.

No processo de filtração da água bruta e nos processos combinados com aplicação de coagulante, verifica-se que houve remoção na sua totalidade de coliformes totais na água final obtida. Assim, pode-se dizer que a membrana se comportou como um método de desinfecção física para microrganismos. $\mathrm{O} \mathrm{pH}$ da água tratada não sofreu grandes alterações, pois segundo Ndabigengesere e Narasiah (1996), dentre as vantagens relacionadas ao uso de Moringa oleifera no tratamento de água, cita-se o fato de tal coagulante não promover alterações significativas no $\mathrm{pH}$ da água tratada, o que resulta em uma maior facilidade operacional e redução de custos tendo em vista que não há necessidade de correção do $\mathrm{pH}$.

Franco et al., (2010) constataram que a aplicação de coagulante a base de Moringa oleifera em um sistema piloto de filtração em múltiplas etapas culminou em maiores eficiências de remoção de turbidez do que a filtração sem adição de coagulante. Em geral, pode-se afirmar que tanto o processo combinado, quanto o processo apenas de ultrafiltração foram eficientes e produziram uma água tratada de boa qualidade. Sendo assim, deve-se avaliar o entupimento da membrana para obter maiores conclusões. 
A Figura 1 apresenta o comportamento do fluxo de permeado obtido no ensaio de filtração e no processo combinado com a membrana de 0,1 e $0,2 \mu \mathrm{m}$ - monocanal, em temperatura de $25^{\circ} \mathrm{C}$, a 1 bar e turbidez inicial de $150 \mathrm{uT}$.

(a)

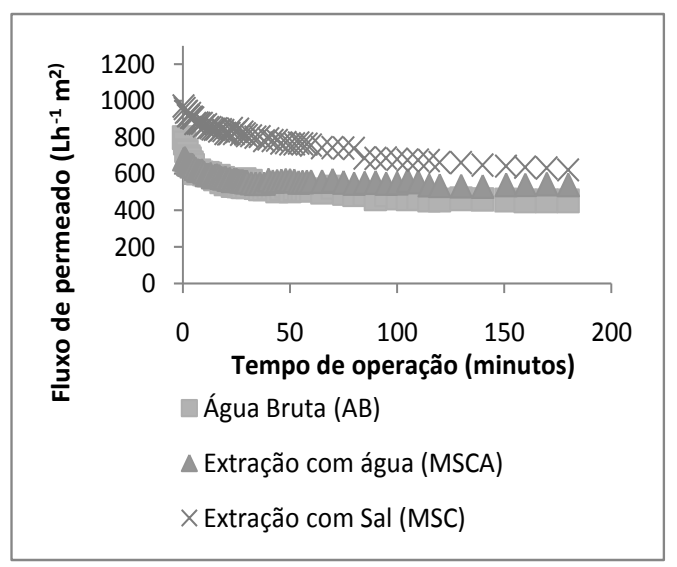

(b)

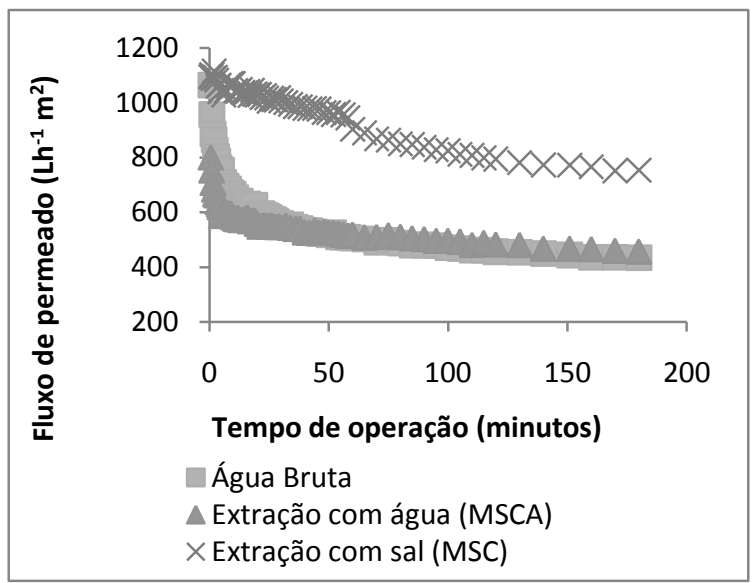

Figura 1. Fluxo de permeado em função do tempo de operação: (a) membrana de $0,1 \mu \mathrm{m}-$ monocanal; (b) membrana de $0,2 \mu \mathrm{m}$ - monocanal.

Os fluxos permeados diminuem com o tempo durante o processamento até atingirem um patamar onde permanecem constantes, chamado de fluxo estabilizado. Em todos os ensaios realizados o fluxo permeado diminuiu com o tempo de processamento (Figura 1). Na membrana de 0,1 $\mu \mathrm{m}$ pode-se observar que o entupimento no ensaio com água bruta (AB) em aproximadamente 180 minutos, sem a adição de coagulante foi de $92,71 \%$ e de $90,06 \%$ quando utilizado MSCA (extração com água) e $86,43 \%$ para MSC (extração com solução salina).

Assim, observa-se uma redução no entupimento quando se utiliza as soluções coagulantes em relação à filtração da água bruta, pode-se afirmar que, o processo combinado de coagulação/floculação/filtração apresentou diminuição significativa do entupimento em relação ao processo de ultrafiltração isoladamente. Stopka et al., (2001) afirmam que o declínio do fluxo permeado é o resultado da superposição dos vários mecanismos de "fouling" na membrana.

Li Xu et al. (2002) estudando a combinação de filtração tangencial, floculação e membranas cerâmicas atingiram bons resultados e redução do entupimento na aplicação de coagulante. Fato este, que também foi observado no presente estudo.

Na membrana 0,2 $\mu$ m o "fouling" ou entupimento foi de 92,38 \% para a água bruta (AB), 90,47 \% para utilização da extração com água (MSCA) e 87,04 \% para a extração com sal (MSC) na pressão de 1 bar (Tabela 4). Os maiores entupimentos ocorreram para a filtração da água bruta e também para a água coagulada/floculada com a solução extraída com água (MSCA), assim como ocorreu com a outra membrana estudada. 


\section{9 a 22 de outubro de 2014 \\ Florianópolis/SC}

Tabela 4 - Avaliação do "fouling” da membrana 0,1 e 0,2 $\mu \mathrm{m}$ - monocanal, pressão de 1 bar

\begin{tabular}{l|cc}
\hline Membrana $(\mu \mathrm{m}) / \%$ fouling & $0,1 \mu \mathrm{m}-$ monocanal & $0,2 \mu \mathrm{m}-$ monocanal \\
\hline Água Bruta (AB) & 92,71 & 92,38 \\
Extração com água (MSCA) & 90,06 & 90,47 \\
Extração com sal (MSC) & 86,43 & 87,04 \\
\hline
\end{tabular}

Bouchard et al. (2003), quando estudaram o processo de microfiltração e o processo combinado de coagulação/microfiltração, utilizando como coagulantes o cloreto férrico e o sulfato de alumínio, obtiveram resultados semelhantes, com redução significativa no entupimento da membrana, quando utilizado o processo combinado, como comentado anteriormente, também para águas superficiais com características de cor e turbidez relativamente baixas.

Assim, o processo combinando utilizando como coagulante a solução extraída com sal (MSC) apresentou-se mais eficiente em relação à diminuição do entupimento quando comparado ao processo de microfiltração da água bruta e ao processo combinado utilizando a solução coagulante de Moringa oleifera extraída com água. Cita-se ainda que o comportamento encontrado para esta membrana de $0,2 \mu \mathrm{m}$ foi similar ao encontrado na outra membrana avaliada $(0,1 \mu \mathrm{m})$.

\section{CONCLUSÕES}

Avaliando o processo de filtração com membranas e o processo combinado, nas condições ótimas de coagulação/floculação, observou-se que os processos de ultrafiltração e microfiltração e o processo combinado produziram água tratada dentro do padrão de potabilidade. E ainda que os entupimentos em todos os casos avaliados, foram maiores para o processo de filtração da água bruta do que se comparados ao processo combinado. Por fim, conclui-se que a utilização de soluções coagulantes antes do processo de filtração com membranas, de forma geral, diminui o grau do entupimento. Os menores valores foram encontrados para solução de Moringa Oleifera extraída com sal (KCl 1 Molar), sendo assim este um processo vantajoso para obtenção de água para consumo humano.

\section{REFERÊNCIAS}

APHA, AWWA \& WEF, Standard Methods for the Examination of Water and Wastewater, Clesceri, L. S.; Greenberg, A.E.;. Eaton A.D., 20th Ed., Washington-USA, 1995.

BAWA, L. M., DJANEYE-BOUNDJOU, G., BOUKARI, Y., SANI, A., Coagulação de Algumas Soluções Húmicas Ácidas pela Moringa oleifera Lam: Efeito na Exigência do Cloro, B. Soc. Quím. Eti., v. 15, n. 2, p. 119-129, 2001.

BRASIL, PORTARIA No 2914 MINISTÉRIO DA SAÚDE. Em 12 de dezembro de 2011. Dispõe sobre os procedimentos de controle e de vigilância da qualidade da água para consumo humano e seu padrão de potabilidade. Ministério da Saúde, 2011. 


\section{9 a 22 de outubro de 2014 \\ Florianópolis/SC}

BERGAMASCO, R.; KONRADT-MORAES, L.C.; VIEIRA, M. F.; FAGUNDES-KLEN, M. R.; VIEIRA, A. M. S. Performance of a coagulation-ultrafiltration hybrid process for water supply treatment. Chem. Eng. J, v. 166, n. 2, p. 483-489, 2011.

BOUCHARD, Ç.; SERODES, J.; RAHNI, M.; ELLIS, D.; LAFLAMME, E.; RODRIGUES, M. Membrane fouling in ultrafiltration and coagulation-ultrafiltration of surface water, J. Environ. Eng. Sci. v.2, p.139-148, 2003.

CAMPLESI, D. C. F.; PEREZ, W. E.; SIQUEIRA, E. Q. Remoção de coliformes totais e Escherichia coli utilizando a filtração em múltiplas etapas (FiME) em períodos de alta turbidez da água bruta. $R$. Eletr. Eng. Civil, v. 1, nº 1, p. 14-18, 2010.

CARDOSO, K.C.; BERGAMASCO, R.; COSSICH, E.S.; KONRADT-MORAES, L.C.. Otimização dos tempos de mistura e decantação no processo de coagulação/floculação da água bruta por meio da Moringa oleifera Lam. Acta Sci. Tech., v.30, n.02, p. 193-198, 2008.

CONCEIÇÃO, V. M.; BASSETI, F. J.; BERGAMASCO, R. Desfluoretação de águas subterrâneas a partir da caogulação/floculação com coagulante natural de semente de Moringa Oleífera Lam. Ver. Exacta, v. 6, n. 2, p. 121- 129, 2013.

FRANCO, M.; ARANTES, C.C.; SILVA, G.K.; CHAMLET, J. PATERNIANI, J.E.S.. Uso de coagulante a partir de semente de Moringa oleifera na etapa do pré filtro no sistema de filtração em múltiplas etapas. In CD de anais do VI Congresso International de Ingenieria Agrícola. Chile, 2010.

GUIGUI, C., ROUCH, J.C., DURAND-BOURLIER, L., BONNELYE, V., APTEL, P. Impact of Coagulation Conditions on the in-line Coagulation/UF Process for Drinking Water Production. Els. Sc. - Desalination, v. 147, p. 95-100, 2002.

LI XU; WENPING, L.; SHUQUN, L.; ZHI W.; QIXIN, Z.; YI L. Treating dyieng waste water by ceramic membrane in crossflow microfiltration. Desal, v. 149, p. 199-203, 2002.

MADRONA, G. S. et al. Study of the effect of saline solution on the extraction of the Moringa oleifera seed's active component for water treatment. Water, Air and Soil Pollution. v. 211, p.409415. 2010.

Madrona, G. S., Seolin, V. J, Fagundes-Klen, M. R., Bergamasco, R. The Potential of Different Saline Solution on the Extraction of the Moringa Oleífera Seed's Active Component for Water Treatment. I J Chem. Reac. Eng., v9, A31, 2011.

NKURUNZIZA, T.; NDUWAYEZU, J. B., BANADDA, E.N.; NHAPI, I. The effect of turbidity levels and Moringa oleífera concentration on the effectiveness of coagulation in water treatment. Water Sci. \& Tech, v. 59, n. 8, p. 1551-1558, 2009.

NDABIGENGESERE A., NARASIAH, S. K., Influence of Operating Parameters on Turbidity Removal by Coagulation with Moringa oleifera Seeds, Env. Tech. v. 17, p.1103-1112. 1996.

STOPKA, J., BUGAN, S. G., BROUSSOUS, L., Microfiltration of beer yeast suspensions through stamped ceramic membranes, Sep.Puri. Tech, v. 25, 535-543, 2001. 